\title{
Cytomegalovirus colitis and oesophageal ulceration in the context of AIDS: clinical manifestations and preliminary report of treatment with Foscarnet (phosphonoformate)
}

\author{
J N WEBER, S THOM, I BARRISON, R UNWIN, S FORSTER, D J JEFFRIES, \\ A BOYLSTON, AND A J PINCHING \\ From Praed St Clinic and Depts of Medicine, Virology, Histopathology and Immunology, St Mary's Hospital \\ and Medical School, London
}

SUMMARY Three patients with biopsy diagnosed invasive cytomegalovirus infection of the colon have been seen in the context of the acquired immune deficiency syndrome (AIDS). Cytomegalovirus colitis presented with fever, abdominal distention, bloody diarrhoea and weight loss. Plain abdominal radiographs showed generalised large bowel dilatation in one patient. Cytomegalovirus infection was shown histologically, but the virus could not be cultured from the stool; no other gastrointestinal pathogens could be demonstrated. The patients were treated with a 14 day continuous infusion of Foscarnet $0.08 \mathrm{mg} / \mathrm{kg} / \mathrm{min}$ (phosphonoformate, Astra Pharmaceuticals). One patient showed a partial response to therapy, but the cytomegalovirus colitis relapsed; the second patient had a symptomatic response only and the third patient died of non-cytomegalovirus opportunist infection while on treatment. Two other patients with biopsy proven cytomegalovirus ulceration of the oesophagus were seen, presenting with dysphagia, fever and weight loss. Invasive infection of the gastrointestinal tract with cytomegalovirus is now a major clinical problem in AIDS. Treatment with Foscarnet may be initially effective, but does not eliminate cytomegalovirus infection.

Cytomegalovirus disease in AIDS has been frequently reported as pulmonary and retinal infection. ${ }^{1-1}$ Quinnan et al reported that $100 \%$ of 34 AIDS patients had evidence of cytomegalovirus infection'by viral culture, and that the most common sites were lung, retina, and peripheral blood. ${ }^{+}$ Whereas cytomegalovirus could be found in gastrointestinal tissue at post mortem examination, it was not possible to associate the presence of the virus to specific disease within the gut. Macher et al reported cytomegalovirus viraemia as a frequent preterminal event in AIDS, and postulated that cytomegalovirus pneumonitis and adrenalitis may be the cause of

Address for correspondence: Dr A J Pinching. Dept of Immunology. St Mary’s Hospital Medical School, London W2 IPG.

Received for publication 23 July 1986. death in a proportion of AIDS patients. ${ }^{5}$ In the past, however, the clinical significance of cytomegalovirus within the gut has been controversial, ${ }^{67}$ and it has been held that cytomegalovirus may only superinfect damaged tissue. ${ }^{*}$ Two cases of cytomegalovirus colitis have recently been reported in the context of AIDS, ${ }^{\prime}$ in which histological examination firmly supported the causal relationship between cytomegalovirus and colitis. We report here three cases of cytomegalovirus colitis in the context of AIDS, and two cases of cytomegalovirus associated ulceration of the lower oesophagus. In each case the cytomegalovirus was cultured from at least one site, and cytomegalovirus inclusion bodies were seen on biopsy of the gut, with histological evidence of invasion, as defined by presence of viral inclusions in the muscle layers, with tissue destruction. A wide 
range of gastrointestinal pathogens was sought, including Cryptosporidium spp, but cytomegalovirus was the only pathogen demonstrated. This paper confirms the primary pathogenic role of cytomegalovirus as a cause of colitis and oesophageal ulceration in the context of AIDS.

\section{Methods}

\section{PATIENT SEI.ECTION}

Five patients with biopsy proven symptomatic cytomegalovirus infection of the gut were identified among patients with established AIDS seen at St Mary's Hospital in 1984. The diagnosis of AIDS conformed to the Centres for Disease Control definition for epidemiological purposes, with a major opportunist infection or Kaposi's sarcoma. Each patient had a positive cytomegalovirus culture from urine, but in no instance was it possible to culture cytomegalovirus from the stool or blood. The patients were selected by histological evidence of invasive cytomegalovirus infection in the absence of other gastrointestinal infection. All the patients were seropositive for HTLV-III by membrane immunofluorescence and ELISA assays.

FOSCARNET

Phosphonoformate (Foscarnet) is a potent inhibi-

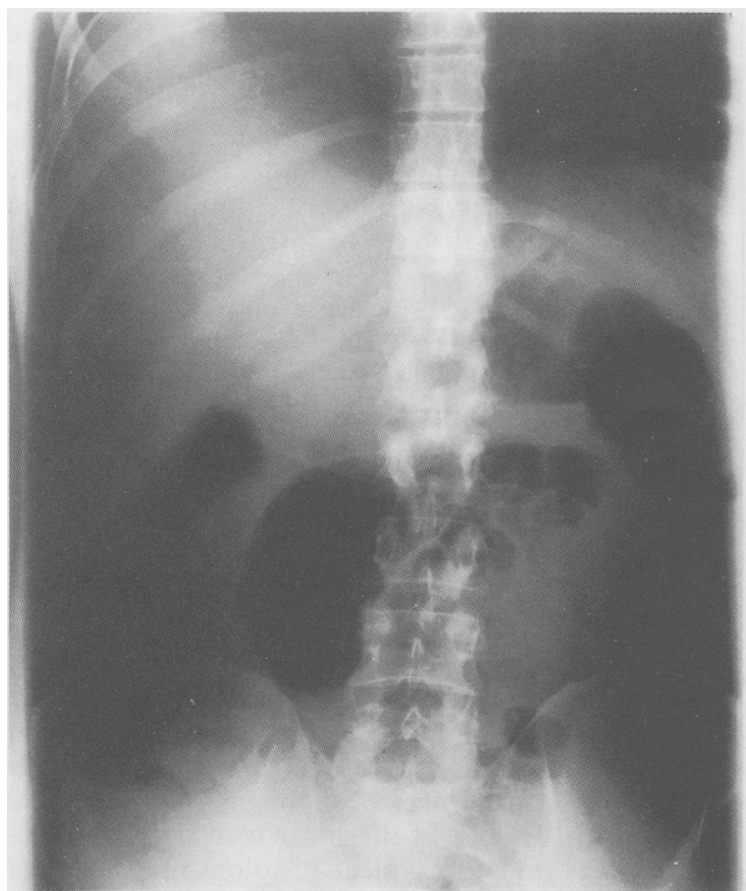

tor of herpesvirus replication." "I The mode of action is probably through non-competitive inhibition of herpes virus induced DNA polymerase, interacting with the enzyme at the pyrophosphate binding site." "I This drug has been thoroughly investigated in animal models, and trials in human herpes virus infections are in progress. ${ }^{2}$ The drug is administered by continuous intravenous infusion, at a rate of $(0 \cdot() 8$ $\mathrm{mg} / \mathrm{kg} / \mathrm{min}$; in each case we used a centrally placed intravenous line for ease of access. Treatment was continued for 14 days if possible. There have been no reported adverse effects, although Foscarnet is known to accumulate in bone, with currently unknown consequences. "Fully informed consent for the administration of a trial drug was obtained from each patient.

\section{Case histories}

CASE 1

A 42 year homosexual man presented with Pneumocystis carinii pneumonia in March 1984. This responded promptly to treatment and the patient was asymptomatic until October 1984, when he was readmitted with a three week history of colicky abdominal pains, watery diarrhoea, tender hepatomegaly and abdominal distension. On examination he was febrile, $38 \cdot 5^{\circ} \mathrm{C}$, and had diffuse abdominal

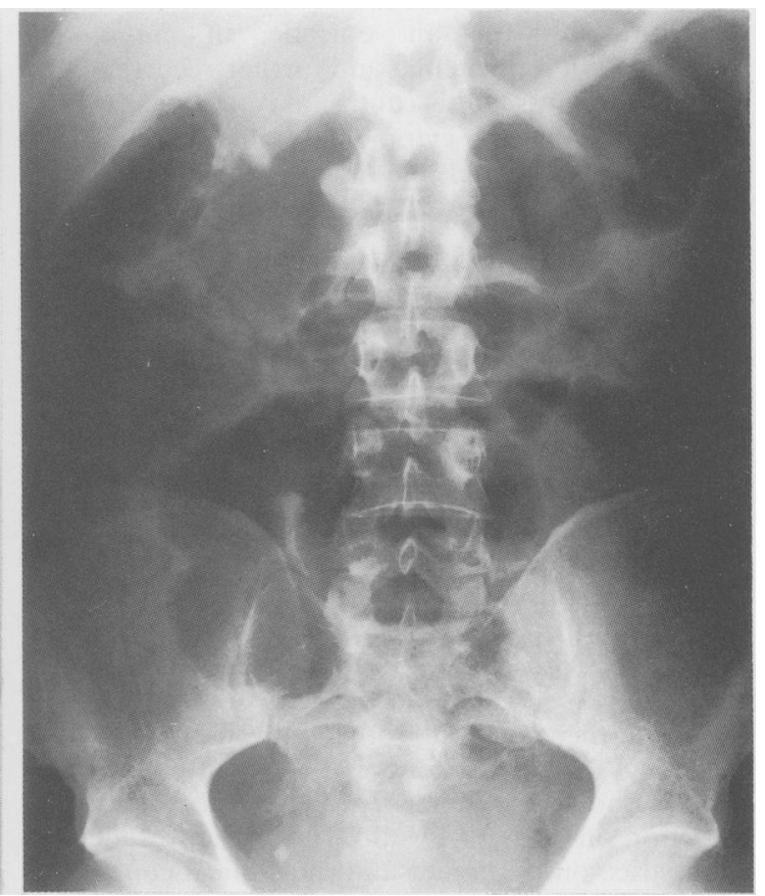

Fig. 1 Erect and supine plain abdominal radiograph of acute episode of cytomegalovirus colitis with large bowel dilationcase 1 . 
tenderness with marked rebound. Plain abdominal radiographs showed generalised large bowel dilatation with fluid levels (Fig. 1); rectal biopsy (Fig. 2) showed the typical changes of invasive cytomegalovirus infection, with inclusion bodies extending to the muscularis layer. Urine culture was positive for cytomegalovirus, but blood and stool were repeatedly negative. Transaminase concentrations were raised, suggesting cytomegalovirus hepatitis. No other pathogens were identified in the stool. The screen included full parasitological, bacteriological, and virological culture and examination. In particular, Cryptosporidiosis was excluded by direct $\mathrm{ZN}$ staining of stool, without concentration.

The patient was initially treated with high dose acyclovir for seven days $(2 \mathrm{~g} /$ day $)$ without response. Foscarnet was then introduced (and acyclovir discontinued), and defervescence occurred within 24 hours, with marked symptomatic improvement, loss of abdominal distension and pain, and resolution of the diarrhoea. This improvement was maintained, and continued after the 14 day course of Foscarnet.

Three weeks after discharge, however, the patient was readmitted with a further episode of cytomegalovirus colitis and fever. Foscarnet was reintroduced for a two week course at the same dosage. There was initial clinical response with defervescence; on the eighth day treatment was discontinued owing to central line sepsis. After this, the clinical course deteriorated with an episode of Salmonella typhimurium septicaemia and further recurrences of cytomegalovirus colitis (untreated); the patient died with disseminated cytomegalovirus infection (as demonstrated by multiple organ needle necrospy) in February, 1985.

\section{CASE 2}

A 50 year bisexual man presented with a nine month history of colicky abdominal pains, diarrhoea, and weight loss. A preliminary diagnosis of malabsorption had been made, but colonoscopic biopsy of the large bowel revealed invasive cytomegalovirus colitis. Stool cultures were repeatedly negative. In addition, there was florid perianal Herpes simplex ulceration, and oral 'hairy' leucoplakia of the tongue.

The patient was treated for Herpes simplex infection with acyclovir $(200 \mathrm{mg} / 5 \times /$ day $)$ and an infusion of Foscarnet was established through a right subclavian line. The patient improved symptomatically, yet there was no objective change in stool frequency or consistency. Post-treatment rectal biopsy showed a persistence of cytomegalovirus infection in the gut wall. The patient failed to defervesce on treatment and the clinical course was complicated by axillary vein thrombosis (related to the central line positioning). The patient subse- quently died of disseminated cytomegalovirus infection (by needle necropsy).

CASE 3

A 37 year homosexual man was well until June 1984, when he developed diarrhoea with bloody stool and oral candidiasis. Shigella flexneri was isolated from stool, and Herpes simplex from perianal ulceration. The symptoms resolved on appropriate treatment (cotrimoxazole and acyclovir). In January 1985, the patient developed abdominal pains, fever and diarrhoea; examination revealed diffuse abdominal tenderness with rebound but no guarding; rectal biopsy revealed cytomegalovirus colitis. The patient was treated with Foscarnet for 10 days, but then developed a large pleural effusion with pneumonitis, and died. Mycobacterium xenopii was subsequently isolated from the pleural fluid. There was no evidence of response to Foscarnet in this patient, but assessment was incomplete owing to the Mycobacterial infection. This patient developed acute renal failure in relation to the disseminated mycobacterial infection unresponsive to therapy.

\section{CASE 4}

A 40 year homosexual man with a nine month history of diarrhoea, malabsorption and weight loss developed a left hemiparesis secondary to a Toxoplasma gondii brain abscess. The cerebral disease improved with Fansidar but the diarrhoea did not improve. Oesophagoscopy for persistent dysphagia revealed a large ulcer at the oesophagogastric junction, which was histologically compatible with invasive cytomegalovirus infection. The biopsy was negative for Herpes simplex culture. The patient was treated with 10 days of intravenous Foscarnet and with cotrimoxazole, but died of overwhelming pneumonitis on the 12th day. Cytomegalovirus was isolated in high titre from a post mortem lung needle necropsy, but no Pneumocystis organisms or other pathogens were demonstrated.

\section{CASE 5}

A 58 year homosexual man developed Kaposi's sarcoma limited to the skin, without evidence of gastrointestinal tract involvement, in October 1984. He remained well until January 1985, when he developed severe dysphagia for solids and liquids, had reduced appetite and lost $10 \mathrm{~kg}$ in weight. Barium swallow suggested an ulcer at the lower oesophagus (Fig. 3). Oesophagoscopy revealed a $3 \mathrm{~cm}$ ulcer at the lower oesophagus, with candidal overgrowth. Biopsy of the ulcer wall showed inclusion bodies and the typical histological pattern of cytomegalovirus infection. Herpes simplex virus culture was not performed. Prolonged antifungal 


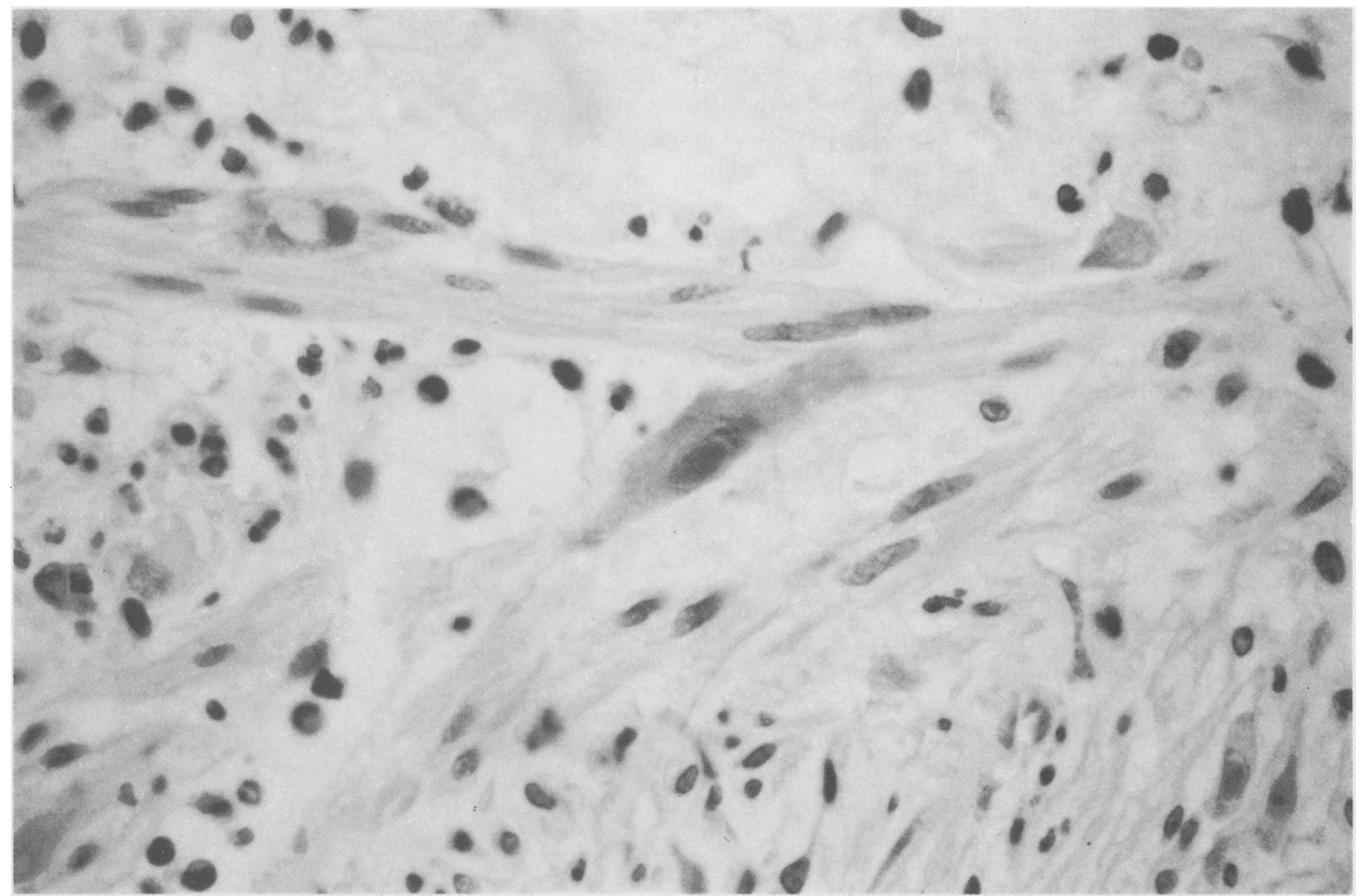

Fig. 2 Rectal biopsy stained with $H$ and E, showing typical cytomegalovirus inclusion in centre of picture, and disruption of the smooth muscle.

treatment with ketoconazole failed to improve the dysphagia, and the patient subsequently entered another trial of treatment for cytomegalovirus infection.

\section{Discussion}

It is common to isolate cytomegalovirus in patients with AIDS, especially from the urine, semen or, less commonly, the buffy layer leucocytes.' The frequent finding of cytomegalovirus in association with other opportunist infections, such as Pneumocystis carinii in the lung, has led to confusion concerning the primary pathogenic role of cytomegalovirus in AIDS.' The severity of cytomegalovirus as an opportunist pathogen has often been overlooked, and hitherto this infection has been untreatable. It is our opinion that diseases directly caused by cytomegalovirus in AIDS (pneumonitis, colitis, oesophagitis, retinitis, encephalitis) are late phenomena in AIDS, and imply a poor prognosis. It is still unclear whether the poor prognosis is a result of the cytomegalovirus-induced immunosuppression added to that of AIDS or a consequence of the profound immunosuppression that leads to the development of cytomegalovirus-related disease. ${ }^{1314}$

We selected for treatment cases of symptomatic cytomegalovirus infection of the gastrointestinal system associated with proven invasion on biopsy. In every case no other pathogen was isolated from the gut to account for the symptoms. The three cases of cytomegalovirus colitis all presented with a similar clinical picture: watery diarrhoea (with bloody stool in two cases), abdominal pain and distension with marked tenderness and rebound, weight loss and fever. In one case (case 1) there were recurrent bouts of large bowel dilatation resembling toxic mega colon, with marked rebound tenderness and guarding on abdominal palpation. These clinical features are similar to the two cases reported by Meiselman et al." The report of progression of cytomegalovirus colitis to necrotising colitis with large bowel perforation confirms the serious nature of this disease." There would now seem to be little doubt that cytomegalovirus infection in the context of the profound immunosuppression seen in AIDS may lead to frank ulceration of the gut. The high prevalence of cytomegalovirus infection in homosexual 
men may be a major factor in the emergence of this new gut manifestation of cytomegalovirus disease. ${ }^{16}$ in

Owing to the poor prognosis of invasive cytomegalovirus disease, these cases were treated with Foscarnet on a trial basis. In case 1, there was a prompt response to Foscarnet on two occasions. The response was not sustained, however, with relapse of cytomegalovirus colitis after each treatment. Patient 2 improved symptomatically, yet biopsy showed persistence of cytomegalovirus in the bowel wall suggestive of progression, and the patient died with disseminated cytomegalovirus some weeks later; there was no objective response to Foscarnet in this case. The responses in cases 3 and 4 are not readily interpretable owing to supervening infection. These latter cases reveal the difficulty of interpretation of infection in AIDS if more than one organism is present, as is so frequently the case by the time that cytomegalovirus infection occurs. Cytomegalovirus viruria was, however, not eradicated in any patient, and notably cytomegalovirus was still present in high titre in the lung of patient 4 , who died while on Foscarnet infusion. It is noteworthy that cytomegalovirus could not be cultured from the stool in any of these patients, although all patients had cytomegalovirus viruria; this may reflect the difficulty of viral culture on stool.

These studies suggest that Foscarnet may have a role in the treatment of severe invasive cytomegalovirus infection in AIDS, but not universally. Maintenance treatment may be needed in view of the relapse which occurred in case 1 . This is similar to the treatment of Herpes simplex infection in the context of AIDS, where the initial infection may respond to acyclovir, but long term prophylaxis is necessary to prevent recurrence. ${ }^{1710}$ Foscarnet appears to be free of major side effects, and may be suitable for further investigation in the management of cytomegalovirus infection in the immunocompromised. The episode of axillary vein thrombosis would appear to be related to the positioning of the central line, and not to the trial drug.

Sandstrom et al" have recently reported the in vitro inhibition of HTLV-III replication by Foscarnet. There was, however, no evidence of clinical improvement of AIDS in any of these patients. This may be because of the late stage of infection at which these patients were treated in the presence of major opportunist infection.

It is now important to recognise cytomegalovirus infection of the gastrointestinal tract as a major opportunist infection in AIDS. The presence of cytomegalovirus inclusions on gut biopsy, in an appropriately symptomatic patient, should be considered evidence of cytomegalovirus disease in the context of AIDS, especially in the absence of other

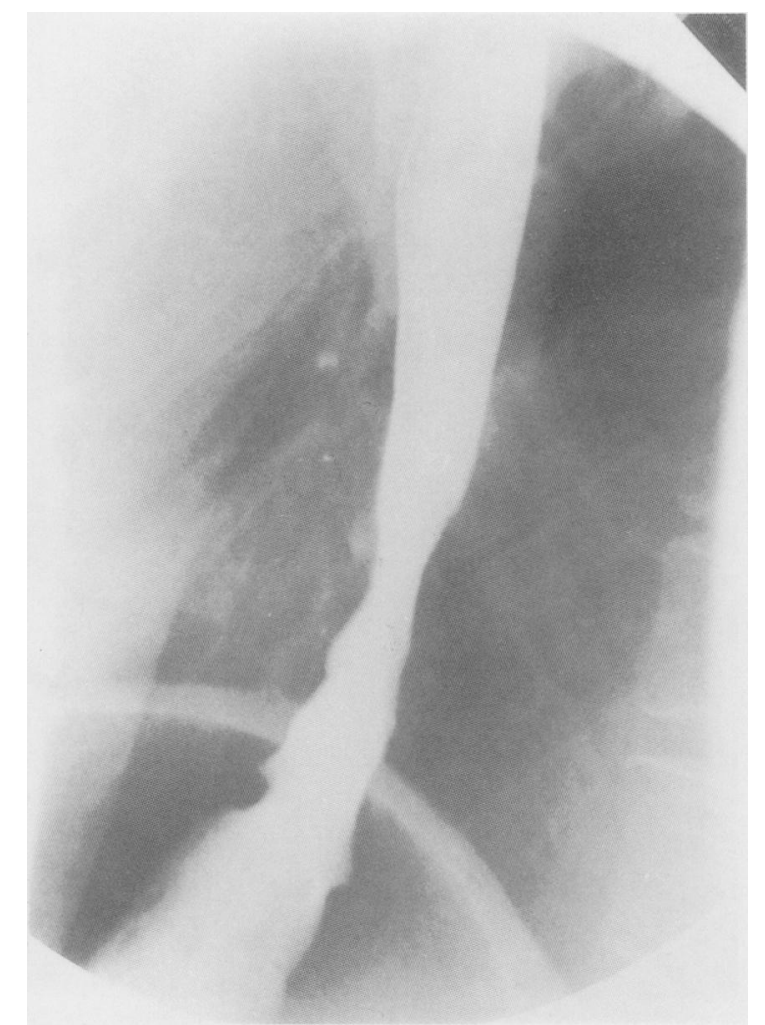

Fig. 3 A barium swallow demonstrating a cytomegalovirus ulcer at the lower third of the oesophagus.

pathogens. The efficacy of Foscarnet and of other new anti-cytomegalovirus agents indicate that cytomegalovirus is now a treatable complication of AIDS.

Reports of the use of dihydroxy propoxymethyl guanine (DHPG) have shown recently remarkable clinical and virological response in cytomegalovirus infection, particularly with cytomegalovirus retinitis. ${ }^{\text {"L.?: }}$ The one patient with cytomegalovirus colitis in these papers only responded symptomatically to DHPG, however, with no biopsy response." Although DHPG may well be a more effective anticytomegalovirus agent for cytomegalovirus retinitis, there are few data on the relative benefits of DHPG versus Foscarnet for cytomegalovirus colitis.

We should like to thank Drs D Silk, A Mee, and B Morson for referring the patients, and Dr Alex Chanas of Astra Pharmaceuticals for supplying Foscarnet and for continuing advice; Ms D Flowers and the Dept of Virology for virological support; Sister Anthony and the nursing staff of AlmrothWright ward for their dedicated work. 


\section{References}

1 Quinnan GV, Masur H, Rook AH, et al. Herpes virus infections in AIDS. JAMA 1984; 252: 72-7.

2 Stover DE, White DA, Romano PA, Gellene RA, Robeson WA. Spectrum of pulmonary disease associated with AIDS. Am J Med 1985; 78: 429-37.

3 Hawley DA, Schefer JF, Schulz DM, Muller J. CMV encephalitis in AIDS. Am J Clin Pathol 1983; 80: 874-7.

4 Freeman WR, Lerner CW, Mines JA, et al. A prospective study of the ophthalmologic findings in AIDS. Am J Ophthalmol 1984; 97: 133-42.

5 Macher AM, Reichert CM, Strauss SE, et al. Death in the AIDS patient - role of CMV. [Letter]. $N$ Engl J Med 1983; 309: 1454.

6 Goodman ZD, Boitnott JK, Yardley JM. Perforations of the colon associated with CMV infection. Dig Dis Sci 1979; 24: 376-80.

7 Cooper HS, Raffensperger EC, Jonas L. CMV inclusion in patients with ulcerative colitis and toxic dilatation requiring colonic resection. Gastroenterology 1977; 72: 1253-6.

8 Foucar E, Mukai K, Foucar K, Sutherland DE, Van Buren DC. Colonic ulceration in lethal CMV infection. Am J Clin Pathol 1981; 76: 788-801.

9 Meiselman MS, Cello JP, Margaretten W. Cytomegalovirus colitis. Gastroenterology 1985; 88: 171-5.

10 Oberg B. Phosphonoformate. Pharmacol Ther 1979; 19: 387.

11 Dolin R. Antiviral chemotherapy and prophylaxis. Science 1985; 227: 1296-303.

12 Wallin J, Lernestedt J-O, Lycke E. Treatment of genital herpes simplex with phosphonoformate. Ex Med Int Congr Ser 1982; 571: 137.

13 Lange M, Klein EB, Kornfield H, Cooper EZ, Grieco
MH. CMV isolation from healthy homosexual men. JAMA 1984; 252: 1908-10.

14 Mintz L, Drew WL, Miner RC, Braff EH. CMV infections in homosexual men. Ann Intern Med 1983; 99: 326-9.

15 Biggar RJ, Andersen HK, Ebbesen P, et al. Seminal fluid excretion of CMV related to immunosuppression in homosexual men. Br Med J 1983; 286: 2010-12.

16 Sonnabend J, Witkin SS, Purtilo DT. AIDS, opportunist infections and malignancies in homosexual men. JAMA 1983; 249: 2370-4.

17 Straus SE, Seidlin M, Takiff H, Jacobs D, Bowen D, Smith HA. Oral Acyclovir to suppress recurring herpes simplex virus infection in immunodeficient patients. Ann Intern Med 1984; 100: 522-4.

18 Mindel A, Weller IVD, Faherty A, et al. Prophylactic oral Acyclovir in recurrent genital herpes. Lancet 1984; ii: $57-60$.

19 Sandstrom EG, Kaplan JC, Byington RE, Hirsch MS. Inhibition of human T-cell lymphotropic virus type III in vitro by phosphonoformate. Lancet 1985 ; ii: 1480-2.

20 Masur H, Lane C, Palentine A, et al. Effect of DHPG on serious CMV disease in 8 immunocompromised homosexual men. Ann Intern Med 1986; 104: 41-4.

21 Felsenstein D, D'Amico DJ, Hirsch M, et al. Treatment of CMV retinitis with DHPG. Ann Intern Med 1985; 103: 377-80.

22 Bach MC, Bapwell SP, Knapp NP, Daws KM, Hedstrom PS. DHPG for CMV infections in patients with AIDS. Ann Intern Med 1985; 103: 381-2.

23 Parkin JM, Rowland-Hill CA, Thomson MH, Jeffries DJ, Pinching AJ. Preliminary report of a trial with DHPG for cytomegalovirus infection in patients with the acquired immune deficiency syndrome. In: Staquet $M$, Hemmer R, Baert A, eds. Clinical aspects of AIDS and AIDS-related complex. Oxford: OUP 1986: 195-201. 